> L'utilisation des systèmes nanoparticulaires composés de polymères suscite d'immenses espoirs pour le traitement de maladies graves, notamment les pathologies cancéreuses. Au cours des dernières décennies, différents types de nanoparticules polymères ont été développés (simples, «furtives», ciblées, sensibles à un stimulus endogène ou exogène, et prodrogues) dans le but de proposer de nouvelles thérapies anticancéreuses plus efficaces et plus sûres. Dans cet article, nous passerons brièvement en revue les différentes familles de nanoparticules polymères ainsi que les principales avancées issues de leur utilisation dans le domaine du cancer. <

La délivrance spécifique (ou adressage) de molécules thérapeutiques vers un organe, un tissu ou une cellule malade constitue aujourd'hui un défi majeur pour le traitement des maladies humaines, notamment infectieuses, cancéreuses ou d'origine génétique. En effet, en dépit de leur efficacité intrinsèque, les principes actifs sont souvent confrontés de par leurs caractéristiques physico-chimiques à de nombreux obstacles dès lors que le site d'administration est différent $d u$ site d'action (comme le passage des barrières biologiques, leur dégradation, leur métabolisation, etc.). Des concentrations thérapeutiques efficaces, au niveau du site d'action, peuvent être obtenues mais généralement au prix d'une déperdition importante en principe actif vers d'autres tissus ou cellules qui ne sont pas pathologiques. Ceci occasionne généralement des effets toxiques importants, voire rédhibitoires, conduisant à l'arrêt des traitements.

L'utilisation de systèmes nanoparticulaires (c'est-àdire de dimensions nanométriques) pour transporter les principes actifs offre des solutions potentielles pour le développement de traitements qui soient plus efficaces et moins toxiques pour le patient. En s'appuyant sur des concepts novateurs en chimie et physico-chimie, ainsi que sur le développement de matériaux innovants,

\section{Les nanoparticules polymères pour la délivrance de principes actifs anticancéreux}

Julien Nicolas, Patrick Couvreur

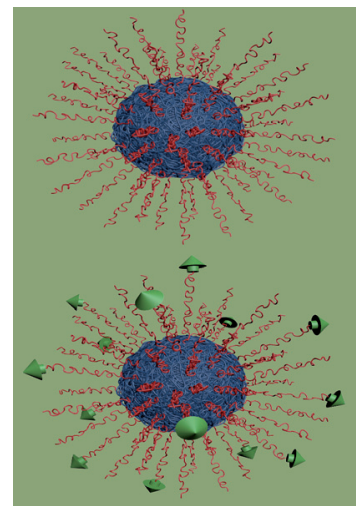

Institut Galien Paris-Sud, UMR CNRS 8612, Université Paris-Sud, Faculté de Pharmacie, 5 , rue Jean-Baptiste Clément, F-92296 Châtenay-Malabry Cedex, France.

julien.nicolas@u-psud.fr

la recherche galénique a permis de concevoir des nanoparticules capables non seulement de protéger le principe actif d'une dégradation précoce mais également d'en contrôler la libération en termes de localisation mais aussi de durée (contrôle spatio-temporel).

Parmi les différents systèmes nanoparticulaires qui ont été développés depuis l'essor de la nanomédecine, les systèmes liposomaux et ceux à base de polymères sont les plus étudiés et les plus prometteurs [1, 2]. L'intérêt pour les polymères, et plus particulièrement les polymères (bio)dégradables (du fait de leur tendance à être excrétés et, pour nombre d'entre eux, de leur non-toxicité), repose sur la facilité de leur synthèse et sur leur très grande diversité de composition, d'architecture et de fonctionnalisation. Parmi les polymères synthétiques les plus utilisés pour le développement de nanoparticules pour la délivrance de principes actifs, nous pouvons citer les polyesters (notamment le polylactide ${ }^{1}$, le polyglycolide et leurs copolymères poly[lactide-coglycolide]) [1], les poly(cyanoacrylate d'alkyle)-PACA [3, 4], ainsi que les poly(acide aminé)s-PAA, ou polypeptides [5] (Figure 1). Selon la nature et la structure des polymères et leur mode de formulation, il est également possible d'obtenir des nanoparticules présentant différentes morphologies: des nanosphères (composées d'un cœur matriciel de polymère), des nanocapsules (composées d'une coque polymère renfermant un cœur huileux ou aqueux), des micelles polymères (qui sont des agrégats de copolymères amphiphiles ${ }^{2}$ possédant

${ }^{1}$ Le lactide est le diester cyclique de l'acide lactique. II est le monomère qui permet de synthétiser le PLA (poly[acide lactique] ou polylactide).

${ }^{2}$ Molécule portant à la fois un groupement hydrophile et un groupement hydrophobe. 


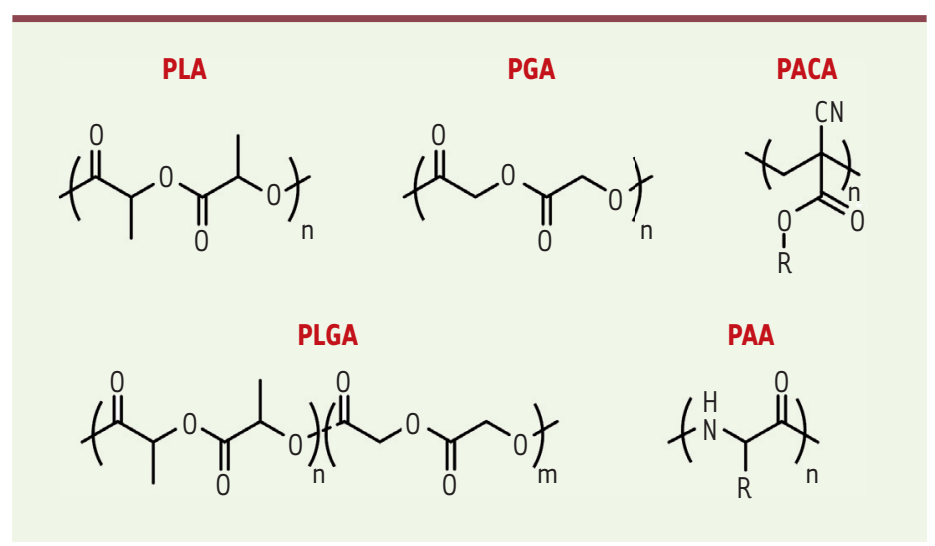

Figure 1. Structure des principaux polymères (bio)dégradables utilisés en nanomédecine. PLA : polylactide; PGA : polyglycolide ; PLGA : poly(lactide-co-glycolide); PACA : poly(cyanoacrylate d'alkyle); PAA : poly(acide aminé).

un bloc hydrophobe et un bloc hydrophile) et des polymersomes (qui sont des vésicules de copolymères à bloc).

Nous aborderons dans cette revue les principaux systèmes nanoparticulaires à base de polymère ainsi que leurs caractéristiques et leurs modes d'action dans le contexte de la thérapie anticancéreuse.

\section{Nanoparticules polymères de première génération}

Lorsqu'elles sont administrées par voie intraveineuse, les nanoparticules polymères de première génération (dont le cœur hydrophobe est simplement stabilisé par un tensioactif) interagissent fortement avec certaines protéines présentes dans le plasma, les opsonines ${ }^{3}$, en raison de leur très grande surface spécifique. Ces dernières étant reconnues par des récepteurs exprimés par des macrophages du système des phagocytes mononucléés (SPM) (au niveau du foie, de la rate et de la moelle osseuse), les nanoparticules recouvertes d'opsonines sont captées, principalement par les cellules de Kupffer (les macrophages du foie). Les nanoparticules sont alors phagocytées et se retrouvent dans les phagolysosomes où elles sont éventuellement dégradées par les enzymes lysosomiales. Cette particularité a cependant été mise à profit pour vectoriser ${ }^{4}$ des molécules thérapeutiques au niveau de ces sites biologiques et ainsi traiter différentes pathologies, notamment cancéreuses. De nombreux travaux ont été consacrés à ce processus de délivrance. Notamment, la distribution hépatique des nanoparticules de première génération a été exploitée dans le traitement de métastases hépatiques. Dans ce cas, comme cela a été démontré avec les nanoparticules de PACA (poly[cyanoacrylate d'alkyle]) [6], il s'agit d'un ciblage indirect : le principe actif anticancéreux est délivré au sein des cellules de Kupffer, qui jouent alors le rôle de réservoir pour le médicament. II peut alors diffuser dans l'ensemble du tissu, notamment vers les cellules cancéreuses.

\footnotetext{
${ }^{3}$ Une opsonine est une molécule (anticorps, composant du complément) qui se fixe sur un antigène (le plus souvent une molécule exprimée à la surface d'une cellule eucaryote ou d'une bactérie) afin d'en faciliter la phagocytose par les cellules immunitaires.

${ }^{4}$ La vectorisation consiste à contrôler la distribution d'un principe actif vers une cible en utilisant un vecteur spécifique de cette dernière.
}

De nombreuses études ont concerné d'autres polymères (bio)dégradables. Plusieurs principes actifs (comme les taxanes, le tamoxifène) ont en effet pu être encapsulés dans des nanoparticules de PLA (polylactide) [7, 8].

La distribution hépatosplénique des nanoparticules polymères de première génération permet également de réduire, dans certains cas, la toxicité du principe actif en les détournant des tissus pour lesquels ils sont toxiques. Ainsi, la toxicité cardiaque de la doxorubicine (Dox) a pu être réduite après son encapsulation dans des nanoparticules de PACA $[9,10]$. Ces résultats ont d'ailleurs conduit à des essais cliniques, actuellement en phase III (Livatag ${ }^{\circledR}$, Onxeo), dans le cadre du traitement du carcinome hépatocellulaire, sixième cancer dans le monde et deuxième cause de mortalité liée à un cancer.

\section{Nanoparticules de seconde génération : des systèmes « furtifs 》}

Tandis que l'accumulation préférentielle dans le foie des nanoparticules de première génération constitue un avantage pour traiter par exemple les métastases hépatiques, elle représente dans de nombreux cas un inconvénient si d'autres organes doivent être ciblés.

Une stratégie, pour contourner efficacement la limitation due à leur opsonisation et leur capture par les cellules de Kupffer, consiste à recouvrir la surface des nanoparticules par du poly(éthylène glycol) (PEG), un polymère hydrophile et flexible, qui empêche les interactions avec les opsonines par répulsion stérique [11]. Ces nanoparticules de seconde génération, dites «P\&Gylées », présentent alors un caractère « furtif » car elles ne sont plus reconnues par les macrophages. Leur durée de résidence vasculaire est donc nettement prolongée. Ces vecteurs ont ainsi une probabilité plus importante de traverser les endothéliums vasculaires présentant une perméabilité accrue, comme ceux du microenvironnement tumoral. II devient alors possible d'effectuer un ciblage en dehors du territoire hépatosplénique, que l'on appelle effet EPR (enhanced permeation and retention) [12].

Le recouvrement des nanoparticules à l'aide de PEG peut être réalisé selon deux approches. La première consiste en une simple adsorption de chaînes de PEG à la surface des nanoparticules. Cependant, il a été démontré que l'adsorption de poloxamers (des tensioactifs macromoléculaires à base de $P E G$ ) n'est que modérément efficace pour prolonger le temps de circulation des nanoparticules, probablement à cause d'une désorption partielle de ces tensioactifs de leur surface [13]. La deuxième approche, qui consiste à lier de manière covalente les chaînes de PEG à la surface des nanoparticules, permet, en revanche, d'éviter 


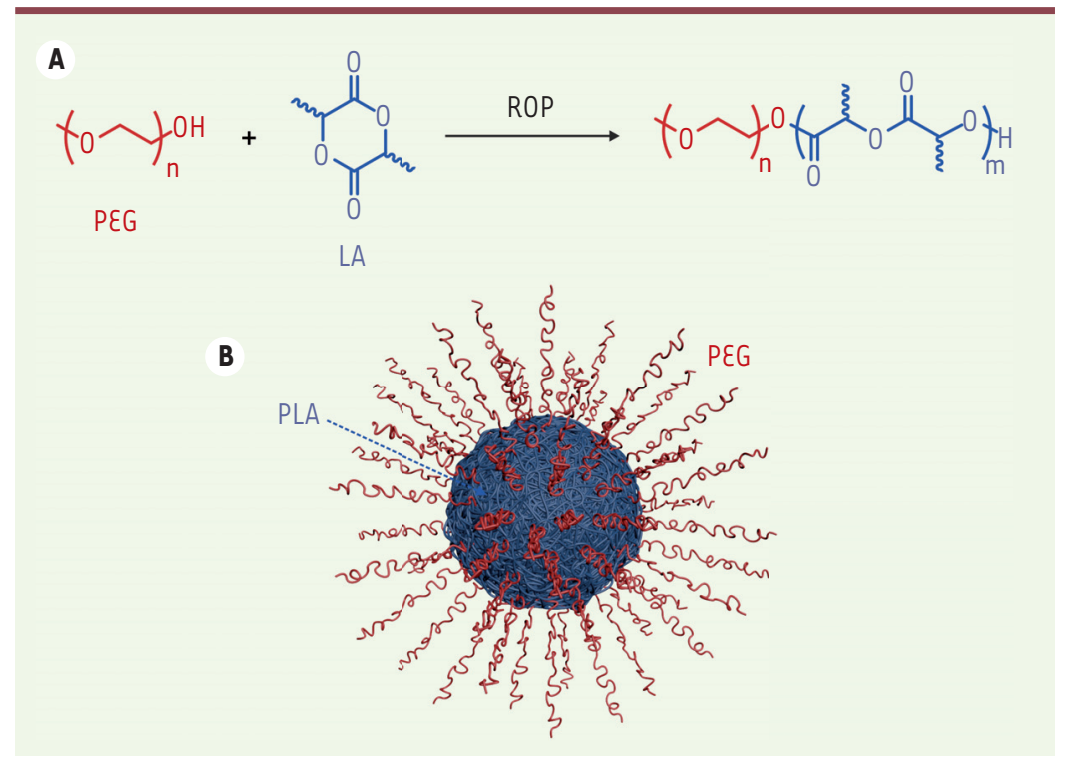

Figure 2. A. Synthèse du copolymère dibloc de PLA- $b$-PEG par polymérisation par ouverture de cycle (radical ring-opening polymerization, ROP). B. Représentation schématique d'une nanoparticule de PLA- $b$-PEG obtenue après formulation. PLA : polylactide ; LA : lactide ; $P \varepsilon G$ : poly(éthylène glycol).

leur désorption et de mieux conserver les propriétés de furtivité des nanoparticules. Le système de référence dans ce domaine est sans conteste le copolymère dibloc de PLA-b-PEG, composé d'un bloc hydrophobe de PLA lié de manière covalente à un bloc hydrophile de PEG. Ce copolymère amphiphile, développé au milieu des années 1990 [13-15], est obtenu par polymérisation par ouverture de cycle (radical ring-opening polymerization, ROP) du lactide amorcée par la chaîne de PEG (Figure 2A). II est capable, lors de sa formulation, de donner des nanoparticules possédant un cœur hydrophobe et biodégradable de PLA, et une couronne stabilisatrice composée de chaînes de PEG conférant la furtivité (Figure 2B). Cette approche a été appliquée à d'autres polymères hydrophobes tels que les poly(acide aminé)s [5] et les PACA [16], permettant également d'augmenter de manière significative le temps de circulation de ces nanoparticules.

En ce qui concerne les nanoparticules PEGylées de PLA ou de poly (acide aminé)s, plusieurs essais cliniques sont actuellement en cours pour le traitement de différents cancers (sein, estomac, œsophage, pancréas, poumon, ovaire) $[17,18]$. On peut citer notamment les micelles polypeptidiques PEGylées de cisplatine et d'oxaliplatine ${ }^{5}$ développées par Nanocarrier (NC-6004 et NC-4016), en essais cliniques de phase I-III, et les micelles polymères chargées en Paclitaxel (Ptx) développées par Nippon Kayaku (NK105), en essais cliniques de phase III [18].

\section{Nanoparticules de troisième génération : des systèmes ciblés}

On parle de vecteurs de troisième génération lorsque les nanoparticules PEGylées sont fonctionnalisées en surface par des ligands (comme des

${ }^{5}$ Des complexes de platine utilisés en chimiothérapie. vitamines, des hydrates de carbone, des peptides, des anticorps, etc.) [2]. Ces systèmes sont capables de reconnaître sélectivement des antigènes ou des récepteurs qui sont surexprimés à la surface de cellules cibles via une interaction de type «clé/serrure » [19]. Ils peuvent donc être guidés vers les cellules qui expriment ces récepteurs, être internalisés et y délivrer leur contenu. Ceci permet d'envisager le ciblage non seulement vers une partie de l'organisme (le foie, le cerveau, etc.), mais également vers un type de cellule bien particulier (les cellules cancéreuses). Leur utilisation en thérapie anticancéreuse est une voie extrêmement prometteuse. Ces nanoparticules ont en effet la particularité non seulement d'augmenter l'efficacité thérapeutique du traitement, en raison de leur accumulation accrue au sein de la tumeur, mais également de diminuer de façon considérable les effets secondaires, en particulier ceux liés à la toxicité des anticancéreux visà-vis des organes et des tissus sains.

Même si la conception des nanoparticules de troisième génération reste encore délicate - notamment eu égard (1) au positionnement d'une quantité suffisante et optimale de ligands à leur surface; (2) à la conservation de leur intégrité chimique et de leur capacité de reconnaissance; et (3) à la conservation de leur stabilité colloïdale et de leur furtivité une fois les ligands positionnés -, cette approche a donné lieu à de très nombreuses études, preuves de concept et essais cliniques [2]. La fonctionnalisation des nanoparticules de PLA- $b$-PEG a notamment été décrite avec différents ligands de ciblage, tels que l'acide folique (vitamine B9), la biotine (vitamine B7), des peptides, des hydrates de carbone, des lectines ${ }^{6}$, de l'albumine, etc. En particulier, in vitro, la capture de nanoparticules fonctionnalisées avec l'acide folique par les cellules cancéreuses est supérieure à celle de nanoparticules dépourvues de la vitamine [20]. Des études réalisées in vivo ont également montré un ciblage actif de tumeurs cancéreuses et une inhibition efficace de la croissance tumorale. Un point important consiste à déterminer la densité de surface de ligands optimale pour que le ciblage soit le plus efficace possible. La modulation de la densité de surface de deux ligands (I'acide folique et la biotine) à la surface de nanoparticules de PEG$b$-PLA, a permis de montrer que des taux de fonctionnalisation de l'ordre de 15 à $20 \%$ permettent d'obtenir

\footnotetext{
${ }^{6}$ Protéines liant les hydrates de carbone.
} 
A

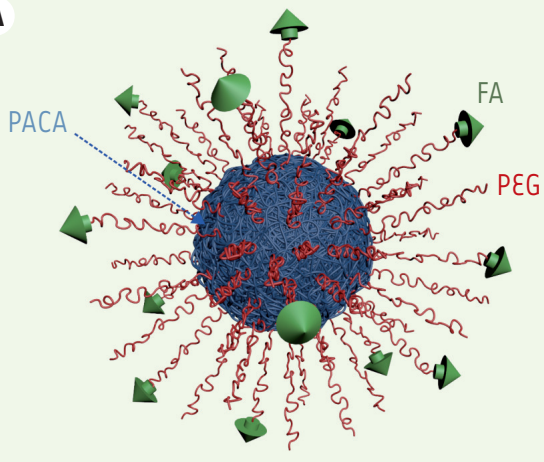

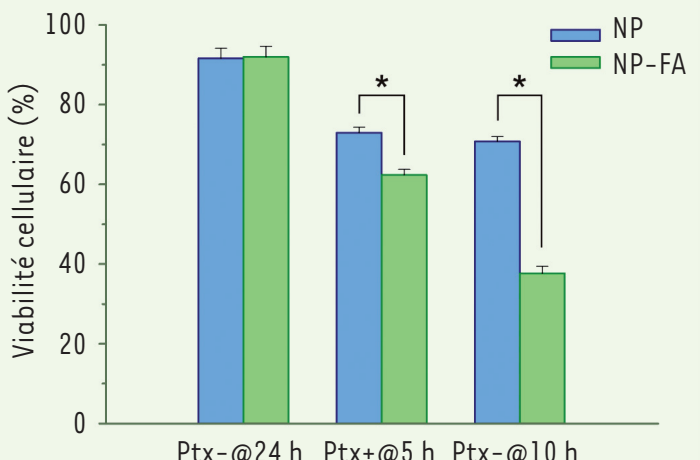

Figure 3. A. Nanoparticules de PACA PEGylées et fonctionnalisées par l'acide folique (FA). B. Viabilité cellulaire de cellules cancéreuses (MCF-7) après incubation avec des nanoparticules de PACA chargées en paclitaxel (Ptx) sans acide folique (NP) ou fonctionnalisées par l'acide folique (NPFA). PACA : poly (cyanoacrylate d'alkyle); PEG : poly(éthylène glycol). *Représente la significativité statistique de la différence.

des affinités maximales vis-à-vis des récepteurs cellulaires [21]. Les nanoparticules de PEG-b-PLA ont également été fonctionnalisées par des molécules de lactose afin d'évaluer, in vitro, leur affinité pour la lectine Ricinus communis [22, 23]. Dans ce cas, les affinités les plus importantes ont été obtenues avec un taux de fonctionnalisation de l'ordre de $80 \%$ (permettant des liaisons trivalentes à la lectine) tandis qu'une fonctionnalisation de $20 \%$ générait principalement des liaisons bivalentes. Des améliorations notables de capture des nanoparticules par les cellules cancéreuses ont été montrées avec d'autres ligands comme le peptide K237 (reconnu spécifiquement par le VEGFR [vascular endothelial growth factor receptor]) [24], les peptides de type RGD (arg-gly-asp, qui ciblent les intégrines $\alpha_{v} \beta_{3}$ surexprimées par les tumeurs cancéreuses lors de la néoangiogenèse [25]) et des aptamères (des oligonucléotides synthétiques [26]). Le système le plus avancé concerne la fonctionnalisation de nanoparticules de PEG$b$-PLA, chargées en docétaxel, par un aptamère capable de cibler le cancer de la prostate. Alors que les essais cliniques (BIND-014, Bind Therapeutics $\operatorname{lnc}$ ) ont été concluants en phase I, ils ont cependant montré une efficacité limitée durant la phase II [27].

La fonctionnalisation de nanoparticules PEGylées polypeptidiques $[5,28]$ et de PACA (Figure 3) par des ligands de ciblage [29, 30] a également montré des efficacités thérapeutiques améliorées dans différents modèles cellulaires. Cependant, aucun système ciblé fondé sur ces polymères n'est à l'heure actuelle évalué en essais cliniques.

\section{Nanoparticules de quatrième génération : des systèmes sensibles à un stimulus}

Même si de nombreux systèmes nanoparticulaires décorés par des ligands de ciblage ont donné des résultats prometteurs in vitro et in vivo en préclinique, ils peinent toujours à montrer un bénéfice thérapeutique indéniable et à s'imposer comme des traitements d'avenir [31]. Ceci semble lié notamment à l'hétérogénéité des cibles biologiques, à la faible accessibilité des tissus malades et des cel- lules cancéreuses, ainsi qu'à une grande variabilité de l'effet EPR. L'élaboration de nanoparticules capables de répondre à des stimulus physiques externes représente donc une voie prometteuse pour accroître leur accumulation dans la tumeur et ainsi augmenter le bénéfice thérapeutique [32]. Les stimulus peuvent être soit exogènes (des variations de température, l'application d'un champ magnétique, de lumière ou de pulses électriques), soit endogènes (des variations de $\mathrm{pH}$, de concentration en enzyme, de différence de potentiel ou d'oxydo-réduction [redox]).

Afin de conférer de telles propriétés aux nanoparticules polymères, deux approches sont envisageables selon la nature du stimulus: utiliser un polymère lui-même sensible au stimulus (de par sa nature, sa structure ou sa composition), ou incorporer au sein des nanoparticules polymères des matériaux sensibles au stimulus, conférant ainsi la sensibilité à l'ensemble de la nanoparticule.

Concernant la première approche, le polymère peut, de par sa nature ou via l'incorporation de fonctions chimiques spécifiques dans sa structure, être rendu sensible à la température, au $\mathrm{pH}$, à la lumière, à une concentration enzymatique, ou à un gradient redox. Par exemple, de nombreux travaux ont été menés sur la synthèse de polymères thermosensibles (dont les propriétés physiques évoluent fortement selon la température). Ces polymères se déclinent en deux groupes différents: (1) ceux possédant une température critique inférieure de solubilité (lower critical solution temperature, LCST) et (2) ceux possédant une température critique supérieure de solubilité (upper critical solution temperature, UCST). Les polymères à LCST sont solubles dans l'eau pour une température inférieure à la LCST et insolubles au-dessus. 


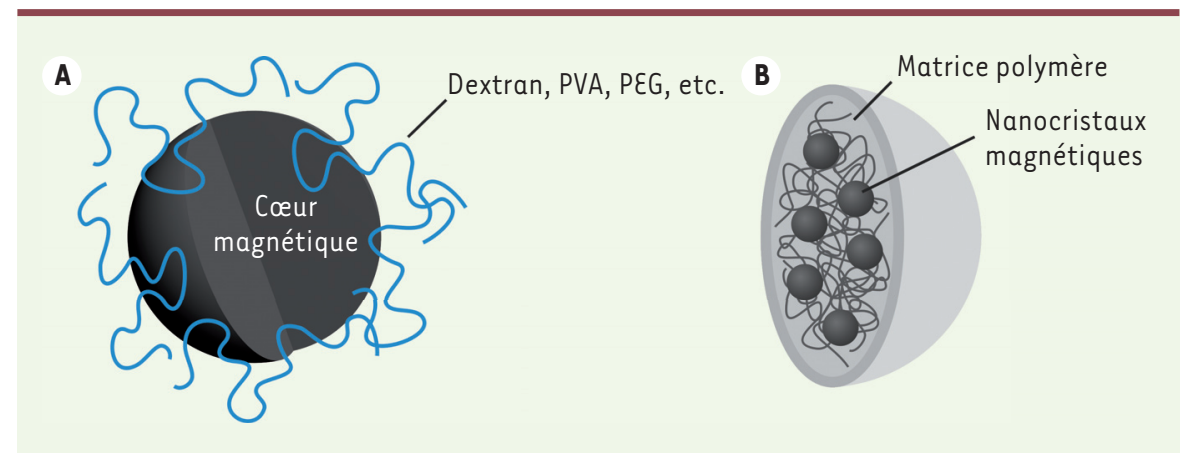

Figure 4. Représentation schématique des nanoparticules magnétiques obtenues par (A) recouvrement d'un cœur magnétique par des chaînes de polymère et (B) par encapsulation de nanocristaux magnétiques au sein d'une matrice polymère. PEG : poly(éthylène glycol) ; PVA : polyvinyl alcohol.

Inversement, les polymères à UCST sont insolubles en-dessous de I'UCST et solubles au-dessus. Cette caractéristique a été avantageusement utilisée pour formuler des nanoparticules recouvertes de poly $(\mathrm{N}$-isopropylacrylamide) (PNIPAAm, un polymère à LCST) [33] et des nanoparticules PEGylées de poly (acrylamide-co-acrylonitrile) (P[AAm-co-AN], un copolymère à UCST) [34] présentant des températures de transition de phase qui sont compatibles avec les systèmes biologiques (entre 30 et $40^{\circ} \mathrm{C}$ ). Ces deux systèmes permettent, sous l'effet d'une légère augmentation locale de la température (hyperthermie modérée), de libérer le principe actif contenu dans la matrice polymère soit par contraction des chaînes de PNIPAAm, soit par solubilisation des chaînes de P(AAmco-AN).

Pour la seconde approche, l'exemple le plus abouti concerne la fabrication de nanoparticules sensibles à l'application d'un champ magnétique [35, 36], I'un des stimulus les plus étudiés. Initialement développée pour les liposomes magnétiques, pour lesquels des essais cliniques avancés sont en cours [32], cette approche a été appliquée avec succès aux nanoparticules polymères magnétiques. Les nanoparticules polymères sont rendues magnétiques soit en recouvrant un cœur magnétique (constitué par exemple de magnétite, $\mathrm{Fe}_{3} \mathrm{O}_{4}$, ou de maghémite, $\mathrm{Fe}_{2} \mathrm{O}_{3}$ ) d'une couche polymère (Figure $4 \mathrm{~A}$ ), soit en encapsulant, au sein d'un cœur polymère, des nanocristaux magnétiques de quelques nanomètres de diamètre composés de magnétite ou de maghémite (Figure 4B). Si le champ magnétique appliqué est continu, il est ainsi possible de guider ces nanoparticules au plus près de la tumeur, simplement en positionnant un aimant à proximité de celleci, permettant alors une accumulation accrue des nanoparticules à ce niveau [37]. Si le champ magnétique est alternatif, ces mêmes nanoparticules vont alors générer de la chaleur dans le milieu environnant et permettre ainsi une hyperthermie sélective des tumeurs, sous réserve de leur accumulation dans celles-ci [38]. Il est également possible de combiner les deux modes d'application du champ magnétique (guidage magnétique puis hyperthermie) afin d'améliorer l'efficacité du traitement. Les nanoparticules d'oxyde de fer permettent de plus de diminuer le signal T2 en imagerie par résonance magnétique (IRM) (relaxation transversale) [50] $(\rightarrow)$, apportant ainsi des informations utiles pour le diagnostic [35]. Plusieurs systèmes à base de

$\rightarrow$ Voir la Synthèse de N. Tsapis, page 18 de ce numéro dextran (Figure 4A) ont d'ailleurs été mis sur le marché comme le Feri$\operatorname{dex}^{\circledast} /$ Endorem $^{\circledast}$, le Resovist ${ }^{\circledast}$ et le Ferumoxtran-10 $0^{\circledast}[18]$.

\section{Nanoparticules de prodrogue polymère}

Les nanoparticules pour la délivrance de principes actifs sont généralement obtenues par encapsulation des principes actifs au sein de la matrice polymère lors de l'étape de formulation et d'auto-assemblage des nanoparticules en solution aqueuse. Cependant, bien qu'étant à l'origine de nombreux résultats prometteurs et essais cliniques (mentionnés précédemment), cette approche possède des limitations importantes comme: (1) le phénomène de burst release (c'est-àdire de libération rapide post-administration d'une fraction significative de principes actifs pouvant entraîner une toxicité); (2) la très faible encapsulation et/ou la cristallisation de certains principes actifs peu miscibles dans la matrice polymère, nécessitant l'utilisation de cosolvants organiques potentiellement toxiques; et (3) le très faible taux de chargement en principes actifs (quelques pourcents) nécessitant l'administration d'une grande quantité de nanoparticules pouvant également conduire à des effets toxiques.

Afin de contourner ces limitations, l'approche «prodrogue», qui consiste à coupler chimiquement le principe actif au polymère, peut être avantageusement utilisée [39]. Trois stratégies sont couramment employées pour synthétiser des prodrogues polymères, qui sont ensuite formulées sous la forme de nanoparticules (Figure 5): (1) le couplage de principes actifs sur un polymère préformé $[5,40]$ (grafting to, Figure $5 \mathrm{~A}$ ) ; (2) le couplage du principe actif sur un motif monomère suivi de l'étape de polymérisation (grafting through, Figure 5B) [41] ; et (3) la méthode du principe actif amorceur qui consiste en l'utilisation d'un principe actif (pré-fonctionnalisé ou non) comme amorceur de polymérisation afin d'obtenir des conjugués principe actif-polymère (drug-initiated ou grafting from, Figure 5C) [42].

Deux exemples représentatifs de la stratégie grafting to sont le poly( $N$-[2-hydroxypropyl] metha- 


\section{A}

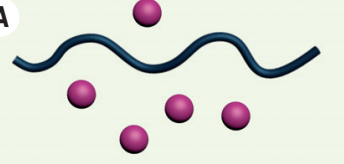

«grafting to »

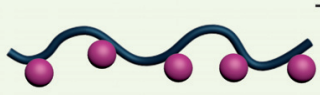

B
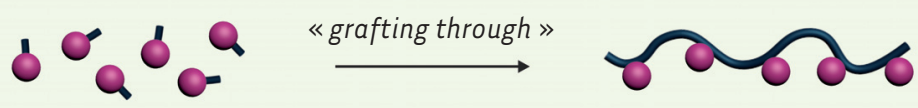

C

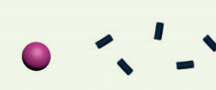

«rug-initiated »
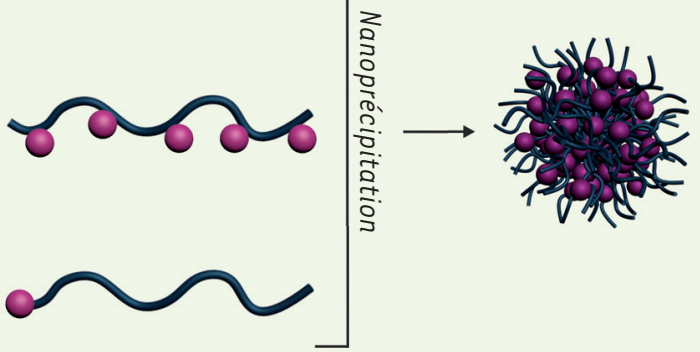

Figure 5. Principales stratégies employées pour synthétiser des nanoparticules de prodrogues polymères. A. Couplage de principes actifs sur un polymère préformé, «grafting to ». B. Couplage du principe actif sur un motif monomère suivi de l'étape de polymérisation, «grafting through ». C. Croissance d'une chaîne de polymère à partir d'un principe actif amorceur, «drug-initiated».

crylamide) (PHPMA) couplé à la doxorubicine [43] et les micelles de polypeptides synthétiques PEGylées également couplées à la doxorubicine ou au paclitaxel [5] qui ont tous les deux conduit à des essais cliniques de phase II. Même si les voies de synthèses proposées pour les deux premières méthodes (grafting to et grafting through) sont relativement complexes, le phénomène de burst release est absent et les taux de chargement en principe actif peuvent être augmentés. La troisième méthode (le principe actif amorceur) permet, quant à elle, de combiner simplicité et efficacité dans la mesure où un nombre minimal d'étapes de synthèse est requis et où des taux de chargement en principe actif élevés (> $30 \%$ massiques) sont obtenus lorsque les chaînes de polymère sont courtes [44-47]. Les conjugués obtenus peuvent également être incorporés dans d'autres systèmes nanoparticulaires [48] ou utilisés comme conjugués solubles si un polymère hydrophile est synthétisé (Figure 5C) [49], offrant ainsi une large gamme de modalités d'action.

\section{Conclusion}

Les différents systèmes nanoparticulaires à base de polymères développés jusqu'à présent ont permis d'effectuer des progrès significatifs dans le traitement du cancer, comme en attestent les nombreux essais cliniques en cours et à des phases avancées. Même si la sophistication de ces systèmes (adjonction de propriétés de ciblage, de sensibilité à un stimulus, etc.) a permis d'obtenir des résultats très prometteurs ainsi que de solides preuves de concept au niveau préclinique, la complexité liée à leur synthèse et à leur transposition d'échelle peut représenter une limitation importante en vue d'éventuels essais cliniques, voire de développements industriels. C'est pourquoi la mise au point de nanoparticules polymères via des stratégies efficaces mais simples à mettre en œuvre apparaissent aujourd'hui primordiales. $\diamond$

\section{SUMMARY}

Polymer nanoparticles for the delivery of anticancer drug Nanocarriers based on polymers are currently attracting much attention to perform efficient drug delivery, especially in cancer therapy. Over the last decades, different kinds of polymer nanoparticulate systems have been developed (e.g., simple, stealth, targeted, stimuli-responsive and prodrug) to propose novel, better and safer cancer therapies. This article will give a brief overview of the different classes of polymer nanoparticles that have been reported and discuss some key achievements deriving from their use in the field of cancer therapy. $\diamond$

\section{LIENS D'INTÉRÊT}

Les auteurs déclarent n'avoir aucun lien d'intérêt concernant les données publiées dans cet article.

\section{RÉFÉRENCES}

1. Soppimath KS, Aminabhavi TM, Kulkarni AR, Rudzinski WE. Biodegradable polymeric nanoparticles as drug delivery devices. J Control Rel $2001 ; 70$ : $1-20$.

2. Nicolas J, Mura S, Brambilla D, et al. Design and functionalization strategies for biodegradable/biocompatible polymer-based nanoparticles applied in targeted drug delivery. Chem Soc Rev 2013 ; 42 : 1147-235.

3. Nicolas J, Couvreur P. Synthesis of poly(alkyl cyanoacrylate)-based colloidal nanomedicines. Wiley Interdiscip Rev Nanomed Nanobiotechnol 2009 ; 1 : 111-27.

4. Vauthier C, Dubernet C, Fattal $\varepsilon$, et al. Poly(alkylcyanoacrylates) as biodegradable materials for biomedical applications. Adv Drug Deliv Rev $2003 ; 55: 519-48$.

5. Bae Y, Kataoka K. Intelligent polymeric micelles from functional poly(ethylene glycol)-poly (amino acid) block copolymers. Adv Drug Deliv Rev 2009; 61:768-84.

6. Chiannilkulchai N, Ammoury N, Caillou B, et al. Hepatic tissue distribution of doxorubicin-loaded nanoparticles after i.v. administration in reticulosarcoma M 5076 metastasis-bearing mice. Cancer Chemother Pharmacol $1990 ; 26: 122-6$. 


\section{RÉFÉRENCES}

7. Krause HJ, Schwarz A, Rohdewald P. Polylactic acid nanoparticles, a colloidal drug delivery system for lipophilic drugs. Int J Pharm $1985 ; 27: 145-55$.

8. Verrecchia T, Spenlehauer G, Bazile DV, et al. Proceedings of the third european symposium on controlled drug deliverynon-stealth (poly[lactic acid/albumin]) and stealth (poly[lactic acidpolyethylene glycol]) nanoparticles as injectable drug carriers. J Control Rel $1995 ; 36: 49-61$.

9. Chiannilkulchai N, Driouich Z, Benoit JP, et al. Doxorubicin-loaded nanoparticles: increased efficiency in murine hepatic metastases. Sel Cancer Ther 1989; $5: 1-11$.

10. Kattan J, Droz JP, Couvreur P, et al. Phase I clinical trial and pharmacokinetic evaluation of doxorubicin carried by polyisohexylcyanoacrylate nanoparticles. Invest New Drugs $1992 ; 10$ : 191-9

11. Veronese FM, Pasut G. PEGylation, successful approach to drug delivery. Drug Discov Today 2005 ; $10: 1451-8$.

12. Iyer AK, Khaled G, Fang J, Maeda H. Exploiting the enhanced permeability and retention effect for tumor targeting. Drug Discov Today $2006 ; 11: 812-8$.

13. Bazile D, Prud'homme C, Bassoullet MT, et al. Stealth Me.PEG-PLA nanoparticles avoid uptake by the mononuclear phagocytes system. J Pharm Sci $1995 ; 84: 493-8$.

14. Gref R, Minamitake $Y$, Peracchia MT, et al. Biodegradable long-circulating polymeric nanospheres. Science $1994 ; 263: 1600-3$.

15. Gref R, Luck $M$, Quellec $P$, et al. Stealth corona-core nanoparticles surface modified by polyethylene glycol (PEG): influences of the corona (PEG chain length and surface density) and of the core composition on phagocytic uptake and plasma protein adsorption. Colloids Surf $B$ Biointerfaces $2000 ; 18: 301-13$.

16. Peracchia MT, Desmaële D, Couvreur P, d'Angelo J. Synthesis of a novel Poly (MePEG cyanoacrylate co-alkyl cyanoacrylate) amphiphilic copolymer for nanoparticle technology. Macromolecules $1997 ; 30: 846-51$

17. Accardo A, Aloj L, Aurilio M, et al. Receptor binding peptides for target-selective delivery of nanoparticles encapsulated drugs. Int J Nanomedicine $2014 ; 9$ : 1537-57.

18. Anselmo AC, Mitragotri S. Nanoparticles in the clinic. BioTM 2016; $1: 10-29$

19. Wang M, Thanou M. Targeting nanoparticles to cancer. Pharmacol Res $2010 ; 62: 90-9$.

20. Tsai HC, Chang WH, Lo CL, et al. Graft and diblock copolymer multifunctional micelles for cancer chemotherapy and imaging. Biomaterials $2010 ; 31: 2293-301$.

21. Mackiewicz N, Nicolas J, Handké N, et al. Precise engineering of multifunctional PEGylated polyester nanoparticles for cancer cell targeting and imaging. Chem Mater $2014 ; 26$ : 1834-47.

22. Yasugi K, Nakamura T, Nagasaki Y, et al. Sugar-installed polymer micelles: synthesis and micellization of Poly(ethylene glycol)-Poly (d,I-lactide) block copolymers having sugar groups at the PEG chain end. Macromolecules 1999 ; 32 : 8024-32.

23. Nagasaki $Y$, Yasugi $K$, Yamamoto $Y$, et al. Sugar-installed block copolymer micelles: their preparation and specific interaction with lectin molecules. Biomacromolecules $2001 ; 2: 1067-70$.

24. Yu DH, Lu Q, Xie J, et al. Peptide-conjugated biodegradable nanoparticles as a carrier to target paclitaxel to tumor neovasculature. Biomaterials $2010 ; 31: 2278-92$.

25. Danhier F, Vroman B, Lecouturier N, et al. Targeting of tumor endothelium by RGD-grafted PLGAnanoparticles loaded with Paclitaxel. J Control Rel 2009 ; $140: 166-73$

26. Farokhzad OC, Cheng J, Teply BA, et al. Targeted nanoparticle-aptamer bioconjugates for cancer chemotherapy in vivo. Proc Natl Acad Sci USA $2006 ; 103: 6315-20$.

27. Von Hoff DD, Mita MM, Ramanathan RK, et al. Phase I study of PSMA-targeted docetaxelcontaining nanoparticle BIND-014 in patients with advanced solid tumors. Clin Cancer Res 2016 $22: 3157-63$

28. Cabral H, Nishiyama N, Kataoka K. Supramolecular nanodevices: from design validation to theranostic nanomedicine. Acc Chem Res 2011 ; 44 : 999-1008.

29. Stella B, Marsaud V, Arpicco S, et al. Biological characterization of folic acid-conjugated poly(H2NPEGCA-Co-HDCA) nanoparticles in cellular models. J Drug Targeting $2007 ; 15: 146-53$.

30. Le Droumaguet B, Nicolas J, Brambilla D, et al. Versatile and efficient targeting from a single nanoparticulate platform: application to cancer and Alzheimer's disease. ACS Nano $2012 ; 6$ : 5866-79.
31. Torrice M. Does nanomedicine have a delivery problem? ACS Cent Sci 2016 ; $2: 434-7$.

32. Mura $S$, Nicolas J, Couvreur P. Stimuli-responsive nanocarriers for drug delivery systems. Nat Mater 2013; 12 : 991-1003.

33. Wei H, Cheng SX, Zhang XZ, Zhuo RX. Thermo-sensitive polymeric micelles based on poly ( $\mathrm{N}$-isopropylacrylamide) as drug carriers. Prog Polym Sci $2009 ; 34: 893-910$

34. Li W, Huang L, Ying $X$, et al. Antitumor drug delivery modulated by a polymeric micelle with an upper critical solution temperature. Angew Chem Int $\varepsilon d 2015$; 54 : 3126-31.

35. Reddy LH, Arias JL, Nicolas J, Couvreur P. Magnetic nanoparticles: design and characterization, toxicity and biocompatibility, pharmaceutical and biomedical applications. Chem Rev 2012 ; 112 : 5818-78.

36. Ulbrich K, Holá K, Šubr V, et al. Targeted drug delivery with polymers and magnetic nanoparticles: covalent and noncovalent approaches, release control, and clinical studies. Chem Rev 2016 ; 116 : 5338-431.

37. Hua MY, Liu HL, Yang HW, et al. The effectiveness of a magnetic nanoparticle-based delivery system for BCNU in the treatment of gliomas. Biomaterials $2011 ; 32$ : 516-27.

38. Hervault A, Thanh NTK. Magnetic nanoparticle-based therapeutic agents for thermo-chemotherapy treatment of cancer. Nanoscale $2014 ; 6$ : 11553-73.

39. Delplace V, Couvreur P, Nicolas J. Recent trends in the design of anticancer polymer prodrug nanocarriers. Polym Chem 2014 ; 5 : 1529-44.

40. Bensaid F, Thillaye du Boullay 0, Amgoune A, et al. Y-shaped mPEG-PLA cabazitaxel conjugates: well-controlled synthesis by organocatalytic approach and self-assembly into interface drug-loaded core-corona nanoparticles. Biomacromolecules 2013 ; 14 : 1189-98.

41. Bertin PA, Smith D, Nguyen ST. High-density doxorubicin-conjugated polymeric nanoparticles via ring-opening metathesis polymerization. Chem Commun (Camb) $2005 ; 30: 3793-5$

42. Nicolas J. Drug-initiated synthesis of polymer prodrugs: combining simplicity and efficacy in drug delivery. Chem Mater 2016; 28 : 1591-606.

43. Kopecek J, Kopecková P. HPMA copolymers: origins, early developments, present, and future. Adv Drug Delivery Rev 2010 ; 62 : 122-49.

44. Harrisson S, Nicolas J, Maksimenko A, et al. Nanoparticles with in vivo anticancer activity from polymer prodrug amphiphiles prepared by living radical polymerization. Angew Chem Int $\varepsilon d 2013$; 52 : 1678-82.

45. Maksimenko A, Bui DT, Desmaële D, et al. Significant tumor growth inhibition from naturally occurring lipid-containing polymer prodrug nanoparticles obtained by the drug-initiated method. Chem Mater 2014 ; $26: 3606-9$.

46. Tong R, Cheng J. Paclitaxel-initiated, controlled polymerization of lactide for the formulation of polymeric nanoparticulate delivery vehicles. Angew Chem Int Ed 2008 ; 47 : 4830-4.

47. Tong R, Cheng J. Controlled synthesis of camptothecin-polylactide conjugates and nanoconjugates. Bioconjugate Chem $2009 ; 21$ : 111-21.

48. Chan JM, Zhang L, Tong $R$, et al. Spatiotemporal controlled delivery of nanoparticles to injured vasculature. Proc Natl Acad Sci USA 2010; 107 : 2213-8.

49. Williams CC, Thang SH, Hantke T, et al. RAFT-derived polymer-drug conjugates: poly(hydroxypropyl methacrylamide) (HPMA)-7-Ethyl-10hydroxycamptothecin (SN-38) conjugates. ChemMedChem $2012 ; 7: 281-91$.

50. Tsapis N. Agents de contraste pour l'imagerie médicale : les exemples de I'IRM et l'ultrasonographie. Med Sci (Paris) 2017 ; 33 : 18-24.

\section{TIRÉS À PART}

J. Nicolas

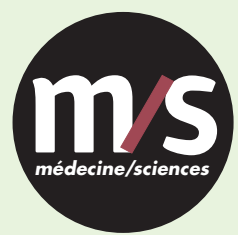

Tarifs d'abonnement $\mathrm{m} / \mathrm{s}-2017$

Abonnez-vous

à médecine/sciences
$>$ Grâce à $\mathrm{m} / \mathrm{s}$, vivez en direct les progrès des sciences biologiques et médicales

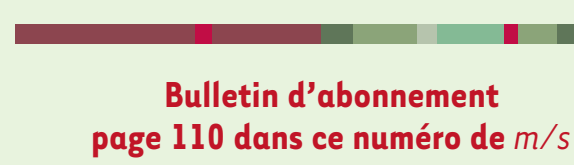

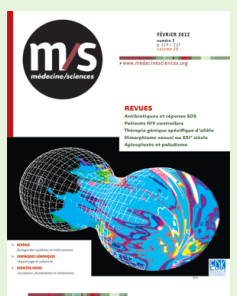

